\title{
Management of cervical deformity in ankylosing spondylitis
}

\author{
Daniel J. Hoh, M.D., ${ }^{1}$ Paul Khoueir, M.D., ${ }^{2}$ and Michael Y. Wang, M.D. ${ }^{3}$ \\ ${ }^{1}$ Department of Neurological Surgery, Keck School of Medicine, University of Southern California, \\ Los Angeles, California; ${ }^{2}$ Department of Neurological Surgery, Université de Montréal, \\ Montréal, Canada; and ${ }^{3}$ Department of Neurological Surgery, Miller School of Medicine, \\ University of Miami, Florida
}

\begin{abstract}
$\checkmark$ Ankylosing spondylitis can lead to severe cervical kyphosis, causing problems with forward vision, swallowing, hygiene, patient functionality, and social outlook. Evaluation of patients with cervical flexion deformity includes assessment of global sagittal balance and chin-brow angle. The primary treatment in extreme disabling cases is surgical correction involving a posterior cervical extension osteotomy, which is a technically demanding procedure with considerable risk of neurological injury. To address the potential complications with extension osteotomy, the authors of several reports have described modifications to the surgical technique. These developments incorporate recent advances in anesthesia, neuromonitoring, and spinal instrumentation. Complications associated with the procedure include subluxation at the osteotomy level, spinal cord injury, radiculopathy, dysphagia, and pseudarthrosis. Although the risks of spinal correction are considerable, extension osteotomy remains an effective treatment modality for patients with disabling cervical flexion deformity. (DOI: 10.3171/FOC/2008/24/1/E9)
\end{abstract}

\section{KEY WORDS • ankylosing spondylitis • cervical kyphosis • chin-on-chest deformity • spinal deformity $\bullet$ surgical correction $\bullet$ surgical technique}

A NKYLOSING spondylitis is an inflammatory condition that can lead to severe pain, disability, spinal fractures, and deformity. Although the underlying cause of AS is unclear, its pathogenesis is an immune-mediated erosion of the joints of the axial skeleton. The spine is particularly targeted, with involvement of the vertebral joints and the intervertebral disc spaces. Lymphocytic infiltration of the disc space leads to degeneration of the junction of the anulus fibrosus and the vertebral endplates. Progressive erosion of these joints is followed by ossification and autofusion, characterized by syndesmophyte formation, widespread ankylosis, and the classic radiographic "bamboo spine" (Fig. 1). Eventually, diffuse osteoporosis transforms the spinal column into a low-density, brittle structure susceptible to fracture with minimal stress. The combination of painful joint inflammation, ankylosis, and osteoporosis predisposes patients with AS to severe spinal deformity.

Ankylosing spondylitic spinal deformity is a result of progressive flexion of the cervical, thoracic, and lumbar spine. Kyphosis develops as patients assume a compensatory flexed posture to unload painful spondylitic facet joints. Autofusion in abnormal sagittal alignment results in a fixed flexion deformity. With global sagittal imbalance, anterior displacement of the patient's center of gravity

\footnotetext{
Abbreviations used in this paper: $\mathrm{AS}=$ ankylosing spondylitis; $\mathrm{CBA}=$ chin-brow angle; $\mathrm{MEP}=$ motor evoked potential; SSEP $=$ somatosensory evoked potential; $\mathrm{VB}=$ vertebral body.
}

leads to osseous remodeling, further kyphosis, and a glacial progression in curvature. Spinal fractures that are undiagnosed or poorly managed also contribute to delayed deformity. The fused osteoporotic spine creates long lever arms, which are particularly susceptible to fracture at junctional levels, even with minor trauma. Cervicothoracic or thoracolumbar fractures that subsequently heal in flexion worsen the overall kyphosis.

Patients with severe spinal deformity can progress to significant disability. Fixed cervical flexion with a chin-onchest deformity leads to difficulty with forward vision, swallowing, hygiene, and social outlook (Fig. 2). The nature of the deformity, the complexity of the underlying disease, and the limited treatment options make managing cervical flexion deformity a challenging problem. Surgical correction of cervical kyphosis is available but is technically demanding and carries the potential risk of devastating neurological injury. The significant psychological and functional impairment of extreme cervical kyphosis, however, mandates appropriate management of patients with AS in whom there are signs of progressive cervical deformity. Although conservative therapy may play a role in early preventative management, treatment of severe cervical kyphosis ultimately requires surgical correction and stabilization.

\section{Initial Evaluation}

The initial evaluation of a patient with AS and a sus- 


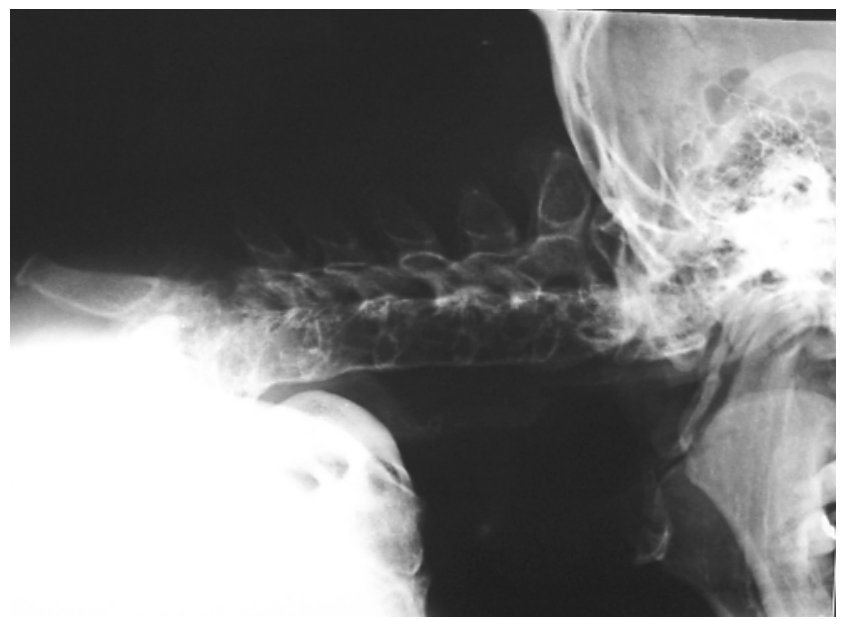

FIG. 1. Lateral radiograph of a patient with AS and a flexion deformity at the cervicothoracic junction. The presence of bridging syndesmophytes creates the "bamboo spine" appearance that is characteristic of AS.

pected cervical deformity is focused on identifying the primary area in need of correction. Examination includes assessing the patient standing upright with hips fully extended as well as in the seated and supine positions. Patients with a primary cervical deformity exhibit persistent cervical flexion despite lying flat, whereas hip or thoracic/lumbar deformities correct in the sitting or supine positions, respectively. If multiple areas require correction, then, generally, the less complicated or morbidity-associated surgery is performed first. Hip deformities are corrected prior to lumbar and thoracic deformities. Surgery for cervical deformity is reserved for patients with only a severe cervical kyphosis or those who have continued sagittal-plane deformity despite appropriate hip, thoracic, and/or lumbar spine correction.

Radiographic evaluation includes a 36-in standing plain $x$-ray film. For cervical deformity, measurement of the $\mathrm{CBA}$ is essential for determining the appropriate degree of correction. The CBA is the angle created by the vertical axis and a line drawn from the chin to the brow (Fig. 3). Normally, the CBA is $0^{\circ}$, but in severe chin-on-chest deformities, this angle can exceed $90^{\circ}$. Surgical treatment of a cervical flexion deformity necessitates meticulous planning to determine the degree of correction. Over correction with iatrogenic cervical extension does not allow the patient to work at a desk or to see the ground in front of him when walking. A final corrected CBA with $\sim 10^{\circ}$ of flexion is generally recommended. ${ }^{9}$ Preoperative planning includes using scissors to cut out a posteriorly based wedge of the selected VB on the x-ray film to determine the appropriate angle of correction necessary. Computed tomography and magnetic resonance imaging provide additional information regarding osseous landmarks for instrumentation or areas of spinal cord impingement or tethering. Flexion/ extension radiographs assess for atlantoaxial instability, which can also occur with AS and present a potential risk of neurologic injury with both intubation and surgery.

Presurgical considerations include obtaining a thorough medical history, as these patients often times are high-risk surgical candidates. Patients with fixed thoracic deformi-

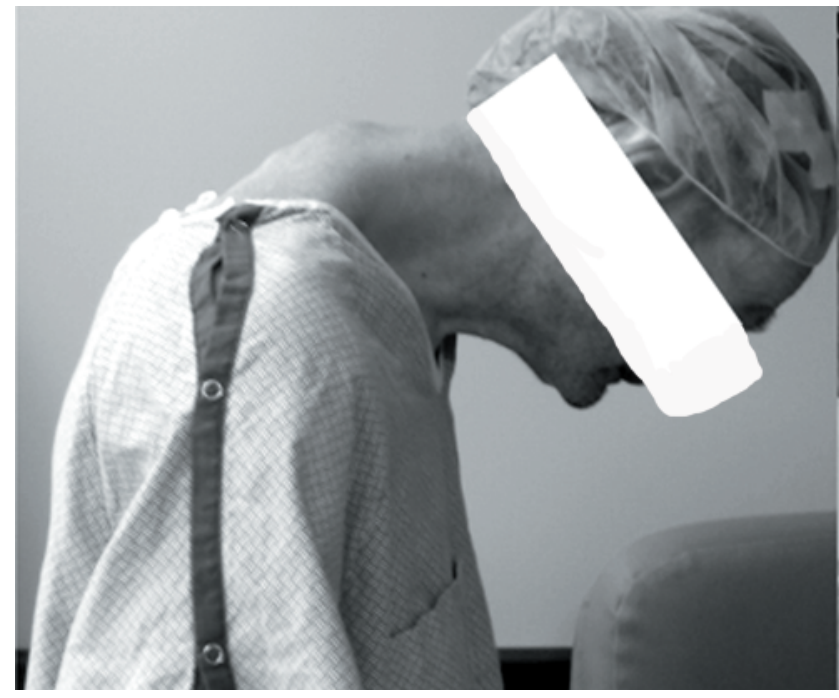

FIG. 2. Photograph of a patient with AS who developed a delayed chin-on-chest deformity after sustaining a lower cervical fracture.

ties may have reduced pulmonary function, which presents greater morbidity with anesthesia and procedures performed in the prone position. Nonsteroidal antiinflammatory agents used to treat chronic pain pose an increased risk of pseudarthrosis. Baseline nutritional assessment is critical because patients with cervical flexion deformity may suffer deficiency due to swallowing difficulties. Perioperative supplementation with tube feedings or parenteral nutrition may be necessary in extreme cases.

\section{Treatment of AS}

Patients who present with only painful arthropathy and mild cervical kyphosis may initially undergo conservative management. Early identification of the initial stages of cervical kyphosis may be responsive to behavior modification, such as sleeping without a pillow under the head, to avoid prolonged neck flexion. Recognition of acute spinal fractures is essential, which may be difficult because of poor imaging capabilities of the cervicothoracic junction. Appropriate brace therapy or stabilization of cervical fractures in neutral alignment, however, may prevent development of a delayed deformity. Closed reduction of a kyphotic deformity with gentle traction may be a viable option in patients with an incompletely ankylosed spine; however, ultimately long-term stabilization with either a halo vest or internal fixation is necessary to ensure fusion in the corrected position. ${ }^{10}$

Surgical correction is reserved for patients with a disabling fixed cervical flexion deformity. Urist ${ }^{19}$ first described the use of cervical extension osteotomy for correction of a chin-on-chest deformity in a patient with AS. This procedure was adapted from Smith-Petersen and colleagues' 16 description of lumbar osteotomies in patients with lumbar flexion deformities. Urist's technique involved removing a posterior wedge of bone from C-7 with subsequent gradual extension of the head and neck to "close" the osteotomy defect and achieve sagittal correction. The inherent nature of the neck deformity made conventional 


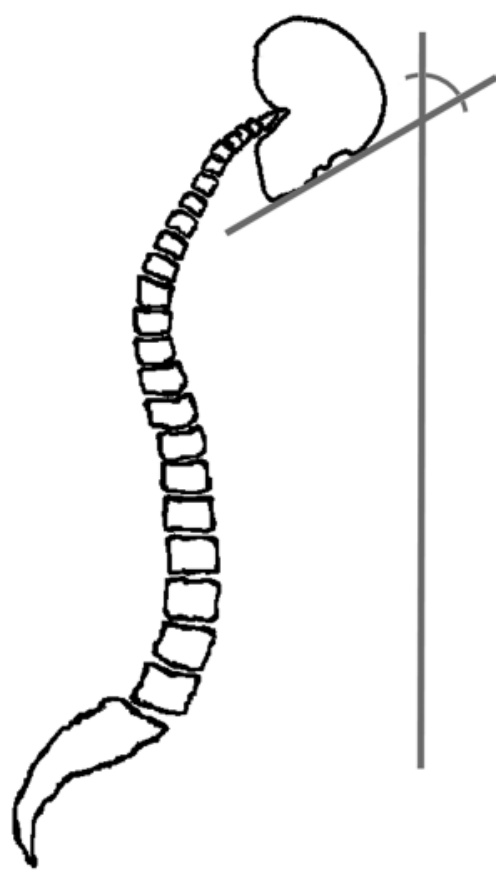

FIG. 3. Illustration demonstrating the measurement of the CBA in severe cervical flexion deformity.

intubation and general anesthesia impossible with standard techniques for that time. As a result, the procedure was performed with the patient awake, after administration of a local anesthetic, and in the sitting position. These anesthesia-related considerations combined with the gravity of performing surgical correction at the level of the cervical spinal cord made the procedure technically demanding with considerable neurological risk. Since then, there have only been a few reports in the literature describing the same technique in a larger series of patients, underscoring the challenging and potentially hazardous nature of the procedure. 1,7, 14 Subsequently, the development and modifications of this technique have largely been detailed through technical case reports and small case series.

\section{Cervical Extension Osteotomy}

Extension osteotomy for correction of cervical flexion deformity was first reported by Urist ${ }^{19}$ in 1958. Later, Simmons ${ }^{15}$ elaborated on the same technique with the first large case series of 42 patients in 1977. Since then, the basic principles of the procedure have largely remained unchanged, except for the later introduction of certain technological advances. In the original description of the procedure, the patient is placed in the sitting position. Anesthesia is administered in the awake patient through a face mask-oxygen and small doses of intravenous anxiolytics and opiates as needed. Precordial Doppler monitoring is performed to evaluate for possible air embolism. With the patient awake, continuous assessment of his/her neurological status is possible. Preoperatively, the patient is fitted with either a plaster thoracic body cast with incorporated halo supports or, more recently, with a removable plastic halo vest. ${ }^{14}$ In the operating room, the halo ring is affixed to the patient's head with cranial pins and initially placed on gentle traction $(<10 \mathrm{lb})$ in direct line with the neck to stabilize the head.

Lidocaine is injected locally in the region overlying the posterior cervicothoracic junction. A vertical midline incision is followed by standard subperiosteal exposure of the C6-T1 spinous processes and laminae, extending laterally to the facet joints. The area of bone removal consists of the full extent of the C-7 lamina, plus the inferior laminar edge of C-6 and the superior laminar edge of T-1 (Fig. 4A). The C-7 level is selected for osteotomy based on several factors. At C-7, the vertebral artery remains anterior to the transverse process and generally does not enter the confines of the tranverse foramen until C-6. Therefore, cervical exten-
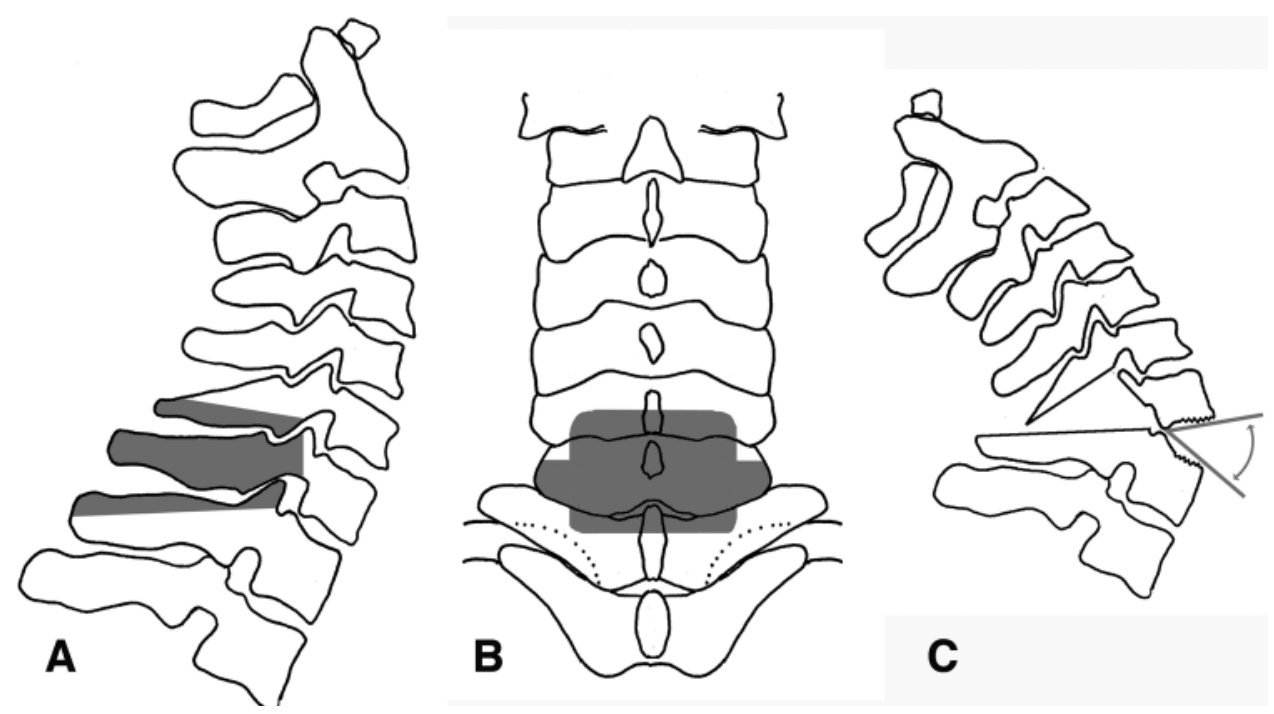

FIG. 4. Illustrations demonstrating a lateral view of the extent of osseous resection for a C7-T1 extension osteotomy (A), a posterior view of the extent of osseous resection for a C7-T1 osteotomy (B), and a lateral view of the angle of correction achieved after an extension osteotomy $(\mathrm{C})$. 
sion at C-7 avoids kinking of the vertebral artery and compromising bilateral cerebral blood flow. The spinal canal is also considerably wider at C-7 compared with the midcervical spine, reducing the likelihood of cord impingement after closure of the osteotomy. Last, in the event of an inadvertent neurological injury, a C-8 spinal cord level preserves significant functional use of the upper extremities and hands. Completion of the osteotomy involves continuing the laminectomy laterally to remove a portion of the C7-T1 facet joint, thereby unroofing the exiting C-8 nerve roots (Fig. 4B). Full decompression of the C-8 nerve roots includes undercutting or completely removing bilateral C7 pedicles to allow for maximum foraminal diameter after correction. Generous removal of the inferior laminar edge of C-6 and superior edge of T-1 prevents osseous impingement of the spinal cord after closure of the osteotomy.

Once the bone resection is complete, the patient receives a short-acting sedative, such as methohexital sodium or propofol, for anesthesia. When the patient is asleep, a surgeon (who leaves the sterile field) manually takes hold of the halo ring and gradually extends the neck. Because of the brittle, ankylosed spine, an intentional opening wedge fracture is created at the anterior columns, often with a clearly audible snap. Osteoclasis of the anterior longitudinal ligament and ventral VB occurs about an internal axis of rotation centered over the posterior longitudinal ligament or dorsal VB (Fig. 4C). During the extension maneuver, a surgeon (who remains in the sterile field) is constantly evaluating the thecal sac to confirm that there is no impingement of the spinal cord. Also, the posterior wedge osteotomy combined with osteoclasis of the anterior columns creates circumferential spinal instability. The surgeon in the operating field is specifically monitoring to ensure that acute subluxation does not occur while extending the neck. Once the desired angle of correction is achieved, the halo ring is attached to the supports and locked into position. Extension effectively closes the posterior osteotomy, with "wrinkling" of the lax dura mater as the posterior length is shortened. The posterolateral margins from C-7 to $\mathrm{T}-1$ are decorticated and local autogenous bone is placed as a graft for fusion. The patient is allowed to awaken from the short-acting anesthetic, and neurological function is assessed. The wound is closed and the patient is maintained in halo immobilization for 4 months postoperatively, followed by a sternal occipital mandibular immobilizer for an additional 2 months. ${ }^{14}$

\section{Technical Modifications}

Urist and Simmons's descriptions ${ }^{15,19}$ of the extension osteotomy remain the standard treatment for correcting a cervical flexion deformity in patients with AS. Several aspects of the procedure, however, have been modified to incorporate advances in anesthesia, neuromonitoring, surgical technique and spinal instrumentation (Table 1). These innovations include utilizing modern anesthesia practices to gain airway access for general anesthesia in patients with severe chin-on-chest deformities. Electrophysiological monitoring has been introduced as a method for evaluating neurological function in patients in whom general anesthesia has been induced. Internal fixation is used for spinal stabilization and as a means for facilitating correction while reducing the risk of acute subluxation during extension. Anterior exposure for an anterior osteotomy has been explored, as well as corrective osteotomies at midcervical levels.

\section{Anesthesia and Neuromonitoring}

Obtaining airway access in patients with severe chin-onchest deformity is challenging. Orotracheal intubation may be impossible in cases in which the patient cannot extend his neck. Risk of neurological injury is also a consideration with any aggressive neck extension in patients with chronic cervical kyphosis. With modern anesthesia techniques, however, awake intubation allows for constant neurological monitoring during induction and insertion of an endotracheal tube. Fiberoptic visualization facilitates inserting a nasotracheal tube to secure airway access in patients with fixed cervical flexion. McMaster ${ }^{9}$ reported successfully performing extension osteotomy in 15 patients in the prone position after induction of general anesthesia. The use of general anesthesia with endotracheal intubation provides several benefits compared with awake anesthesia. With endotracheal intubation, airway access is secured throughout the duration of the procedure. With a secured airway, the procedure can be performed in the prone position, facilitating placement of instrumentation, particularly at the upper thoracic levels, and reduces the risk of air embolism. General anesthesia also ensures patient comfort throughout the procedure.

General anesthesia, however, impairs the ability to monitor neurological function, particularly immediately after the corrective extension maneuver. Spinal cord injury can be caused by an acute translational injury or impingement of the cord from osseous compression upon closure of the osteotomy. McMaster ${ }^{9}$ and others ${ }^{13}$ have described using the Stagnara wake-up test as the gold standard for assessing neurological function after spinal deformity correction. While a wake-up test definitively demonstrates the patient's neurological function, expert anesthesia is required to perform a safe and timely evaluation. The critical time window for reversal of a deficit may elapse while waiting for the patient to recover from anesthesia.

Electrophysiological recording is a noninvasive means for intraoperative monitoring of neurological function. Somatosensory evoked potential and MEP recordings are commonly used for neurophysiological monitoring during many routine and complex spinal procedures. Careful use of anesthetic agents is critical when performing electrophysiological monitoring. Paralytic and nitrous agents are known to impair MEP and SSEP recording, respectively. Propofol infusion during induction with remifentanil and isoflurane for general anesthesia is an acceptable regimen for maintaining anesthesia while allowing MEP and SSEP monitoring. Recording of SSEPs offers the benefit of continuous electrophysiological monitoring, but caution should be used when interpreting this information. Monitoring of SSEPs is used to evaluate primarily the dorsal columns and does not provide information regarding the integrity of the motor pathways. The authors of several reports have described significant postoperative neurological deficits despite having seen normal intraoperative SSEP recordings. ${ }^{2,8}$ 
Cervical deformity in ankylosing spondylitis

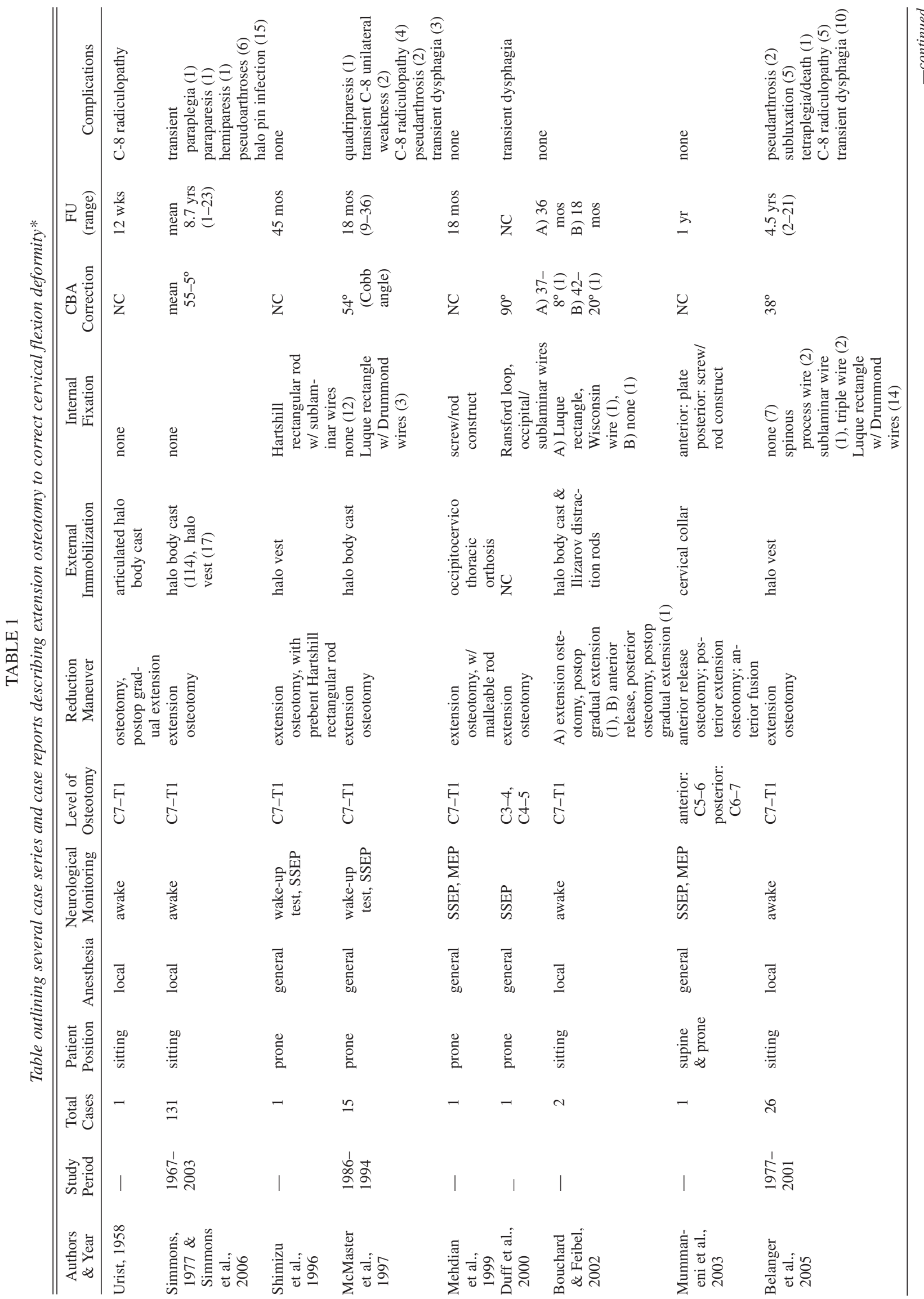




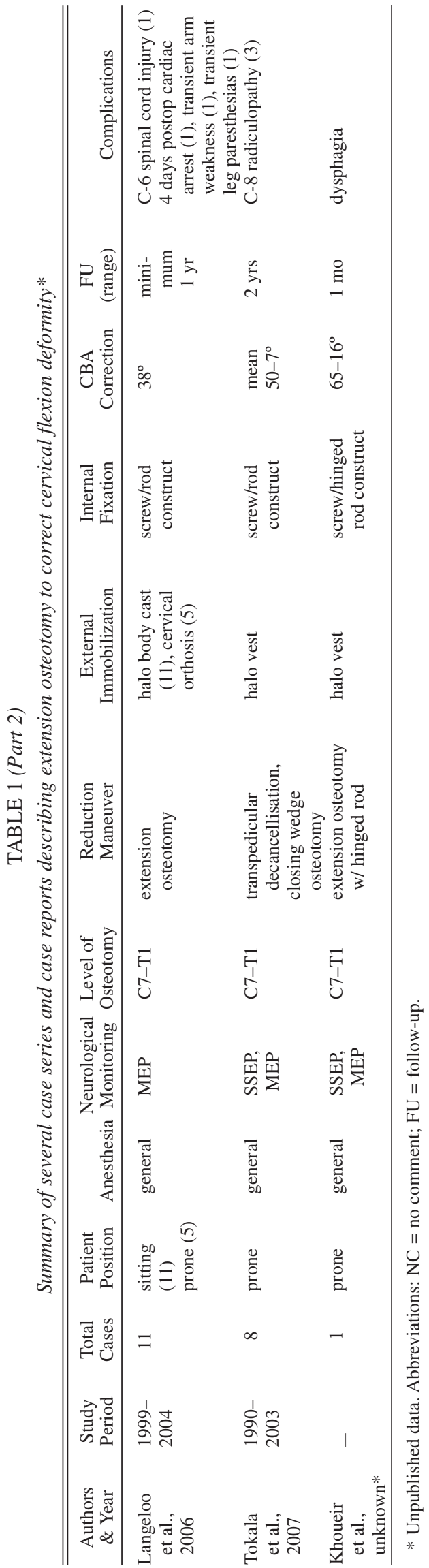

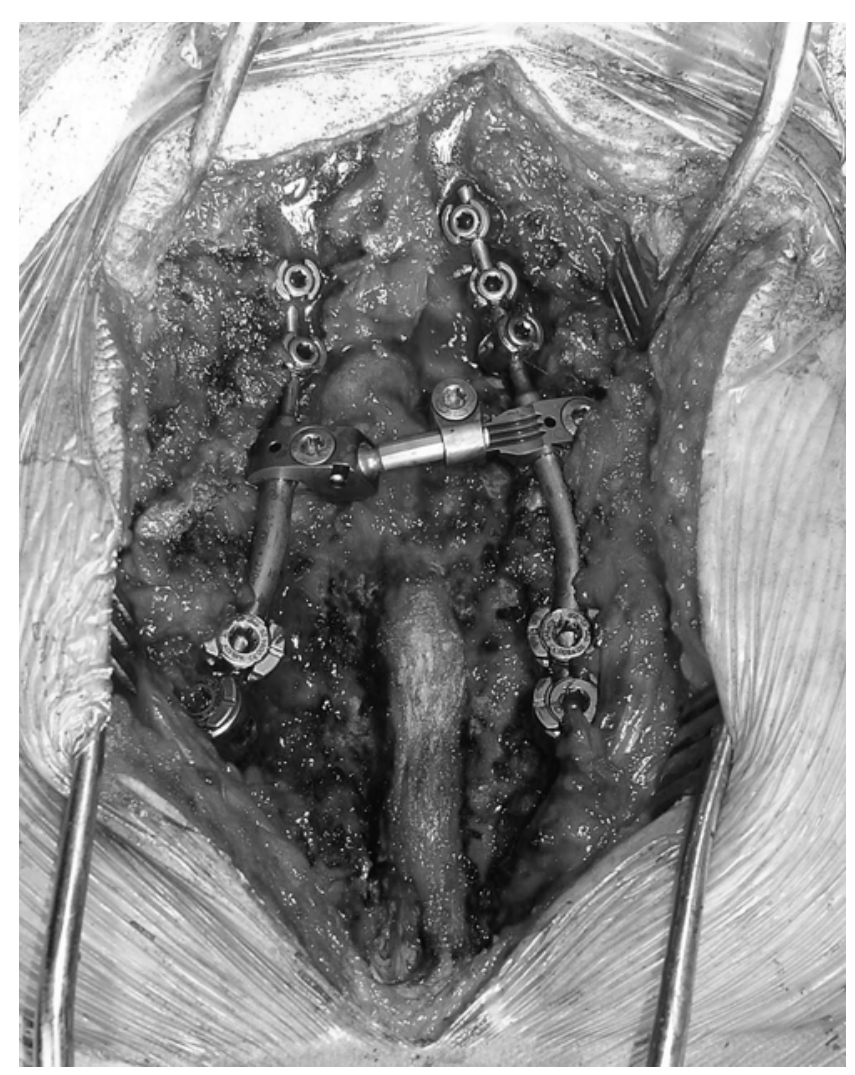

FIG. 5. Intraoperative photograph of posterior spinal instrumentation in which cervical lateral mass and thoracic pedicle screws have been used for internal fixation after an extension osteotomy. Cervical lateral mass screws have been placed at C-3, C-5, and C6 on the left side and at C-4, C-5, and C- 6 on the right, with bilateral thoracic pedicle screws at T-2 and T-3. Titanium rods are affixed to the screws bilaterally with a cross-connector overlying the osteotomy level.

Motor evoked potential recording monitors the corticospinal tracts to evaluate motor function. Langeloo et al. ${ }^{7}$ reported on the use of transcranial electrical stimulated MEP monitoring in 16 patients undergoing cervical extension osteotomy. The extension osteotomy was similar to that described by Urist ${ }^{19}$ and Simmons ${ }^{15}$, but general anesthesia had been induced prior to MEP monitoring. In the report by Langeloo et al., a 20\% decrease in amplitude of MEPs was marked as a significant neurological change. In the event of a decrease in MEPs, technical problems were assessed and hemodynamic parameters were optimized. If the amplitudes remained decreased, then the surgical maneuver immediately preceding the change in MEPs was reversed.

In Langeloo and colleagues' series of 16 patients, ${ }^{7}$ a total of 9 events in 7 patients were detected by changes in MEP amplitude. One patient had an immediate spontaneous recovery of MEP amplitude and did not experience any postoperative motor deficits. In 6 patients, the change in MEPs was immediately preceded by a maneuver in which the neck was extended to correct the deformity. The extension maneuver was reversed in all 6 patients. In 5 of the 6 patients, there was a recovery in MEP amplitude, and nor- 
mal motor function was observed postoperatively. In 1 patient MEPs were not recovered despite the authors reversing the maneuver and performing a secondary anterior decompression the same day. Postoperatively, the patient had a permanent C-6 spinal cord injury. Langeloo et al. also reported using MEP monitoring to evaluate potential pedicle screw violations. It detected 1 malpositioned thoracic pedicle screw, which was subsequently repositioned without complication. Of note, there were no cases of stable intraoperative MEP recordings and a new postoperative motor deficit. Several patients, however, did have postoperative C-8 sensory disturbances, which are not evaluated by MEP monitoring. As a result of these findings, Langeloo et al. advocate using MEP monitoring when performing extension osteotomy in cases requiring general anesthesia.

\section{Internal Fixation}

In a series of 131 patients undergoing extension osteotomy, Simmons et al. ${ }^{14}$ used external immobilization with either a halo cast or halo vest without internal fixation. Halo immobilization without supplemental internal fixation poses the risk of delayed subluxation at the osteotomy site with potential neurological injury or pseudarthrosis. ${ }^{1}$ Reporting on a series of 15 patients, McMaster ${ }^{9}$ treated 12 patients with halo immobilization alone, whereas 3 patients received a halo body cast and underwent internal fixation with a Luque rectangular rod and wiring. In the group of 12 patients without internal fixation, there were 4 cases of postoperative C7-T1 subluxation with 2 patients eventually developing a nonunion and requiring a secondary operation. None of the patients with internal fixation exhibited evidence of subluxation or pseudarthrosis. Belanger et al. ${ }^{1}$ reported on 26 patients who underwent cervical extension osteotomy, of whom 7 received a halo vest alone, whereas 19 underwent some form of internal fixation with wiring. The authors found 5 patients with subluxation at the osteotomy level, all of whom had not undergone internal fixation.

The authors of several reports have described using posterior wiring techniques for internal fixation. ${ }^{4,6,9,13}$ The use of sublaminar wires at thoracic levels poses the risk of spinal cord injury, whereas spinous process wiring provides limited immobilization. Current spinal instrumentation involving lateral mass and pedicle screws provides better fixation and may obviate the need for postoperative halo immobilization. In a review of patients with AS and cervical fractures, Taggard and Traynelis ${ }^{17}$ demonstrated that posterior cervical fixation could be performed safely and with good fusion rates. Long-segment fixation, however, may be necessary to counteract the long lever arms incurred by a rigid ankylosed spine (Fig. 5). Multilevel fixation also improves stability given the generally poor bone quality. ${ }^{5}$ When inserting lateral mass and pedicle screws, however, particular attention must be made to evaluating preoperative radiographs and computed tomography scans because the normal osseous landmarks for screw entry may be misleading. As a result, Taggard and Traynelis recommend using shorter-length screws to reduce the risk of a canal or foraminal violation.

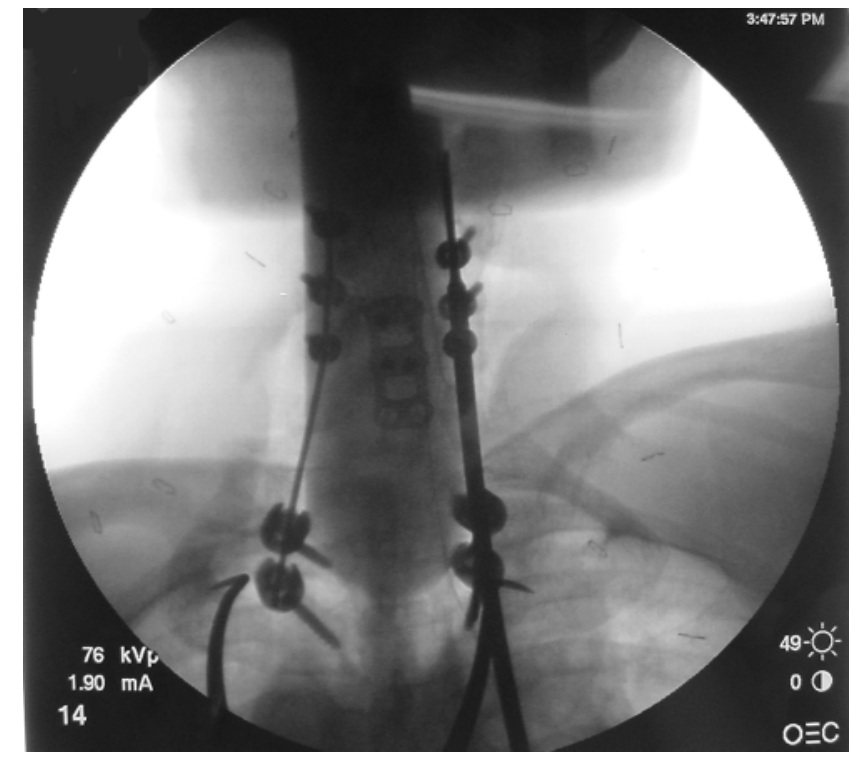

FIG. 6. Intraoperative anteroposterior fluoroscopy image demonstrating a provisional malleable rod (patient's right) and a permanent cervicothoracic titanium rod (patient's left).

\section{Preventing Acute Subluxation}

Cervical correction with extension osteotomy is achieved by manually extending the neck to cause an abrupt osteoclasis of the anterior columns. The resulting opening wedge fracture at the ventral disc space, combined with the posterior osteotomy, creates a significant degree of instability, until either the halo ring or the internal fixation is locked in place. As a result, during manual extension, the patient is at risk for subluxation at C7-T1 with potential for a catastrophic neurological injury. Several techniques for preventing an acute translational injury have been subsequently developed. In Urist's original report ${ }^{19}$ he described performing a gradual postoperative extension by using an articulated halo brace with turnbuckles. Slow correction was achieved with daily elevation of the patient's chin and extension of the neck over the period of a week. Bouchard and Feibel ${ }^{4}$ modified a halo vest with Ilizarov distraction bars and clickers to perform a similar postoperative serial distraction, extension, and spinal correction. Each adjustment was performed while the patient was awake, and without sedation, for continuous neurological evaluation.

With the introduction of spinal instrumentation, several techniques have come to involve various implants to prevent acute translation during extension. Shimizu et al. ${ }^{13}$ reported using sublaminar wires and a prebent Hartshill rectangular loop rod to allow for controlled correction during extension osteotomy. Sublaminar wires are passed beneath C-4, C-5, T-1, and T-2. The rectangular loop rod, bent at an angle of $35^{\circ}$ to approximate the corrected angle, was then attached to the wires at the thoracic levels. The cervical wires are gradually tightened to the rod, with manual repeated releasing and locking of the Mayfield head holder. Final approximation and tightening of the cervical wires to the bent rod corrected the deformity while controlling for both sagittal and lateral translation.

Mehdian et al. ${ }^{11}$ have described using a provisional rod 


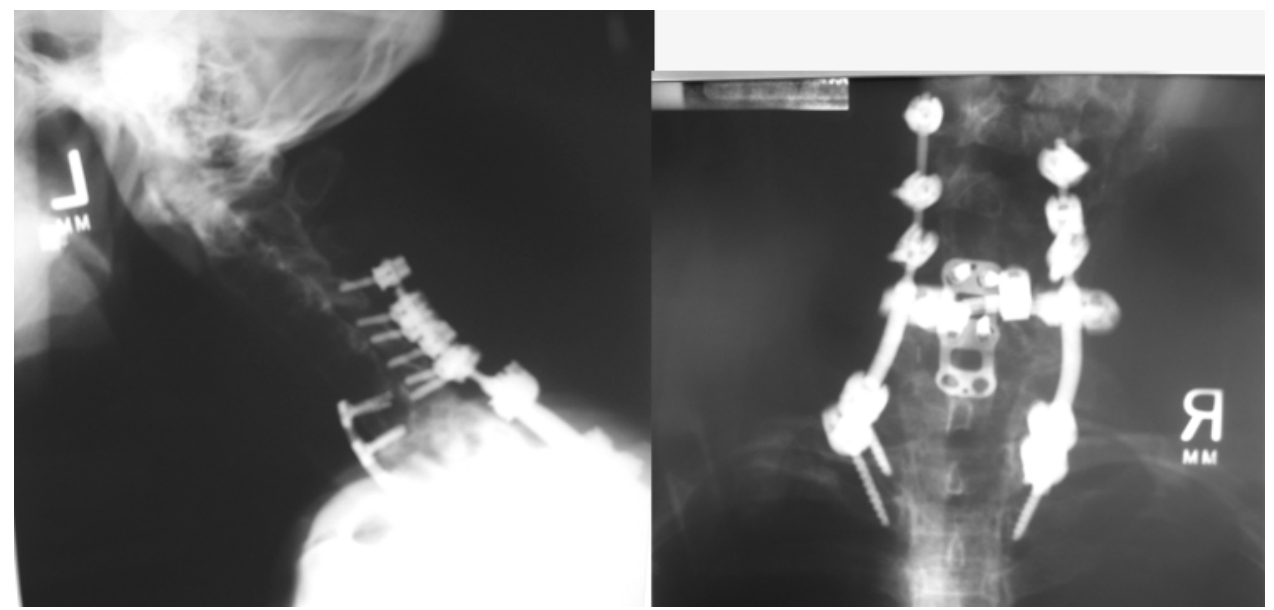

FIG. 7. Postoperative lateral (left) and anteroposterior (right) radiographs obtained after a combined anterior- posterior approach for correction of cervical kyphosis.

threaded through posterior cervical and thoracic screws to prevent acute translation during extension. The patient, in whom general anesthesia has been induced, is positioned prone to allow for placement of cervical lateral mass screws and thoracic pedicle screws. A sterilized malleable intubation stylette is then fixed to the cervical screws and threaded loosely through the thoracic screws heads. With extension of the neck, the malleable rod is guided through the thoracic screws, such that the hinge of sagittal motion occurs through the rod. When the desired correction is achieved, the thoracic screws are tightened to the provisional rod and the Mayfield head holder is resecured. One malleable rod is removed and replaced by a titanium rod bent to match the corrected angle (Fig. 6). After final tightening of the titanium rod to the screws, the contralateral malleable rod is similarly exchanged for a titanium rod. A new variation of this technique involves using a titanium hinged rod (P Khoueir et al., unpublished data). The rod is specially designed with a hinge connector that allows for a large varying degree of angulation. Prior to correction, the portion of the rod superior to the hinge is affixed to cervical lateral mass screws, and the inferior portion of the rod is locked to the thoracic pedicle screws. With neck extension, the sagittal correction occurs about the hinge connectors, preventing both forward and lateral translation. After the definitive corrected position is obtained, the hinge connector is secured with an inner locking screw, creating a rigid rod for permament internal fixation.

The danger of subluxation occurs due to the abrupt opening wedge fracture of the ventral VB. To circumvent this from occurring, Tokala et al. ${ }^{18}$ have performed a C-7 decancellization closing wedge osteotomy to correct cervical flexion deformities. A posteriorly based wedge osteotomy overlying C-7 is resected, similar to that described by Urist ${ }^{19}$ and Simmons. ${ }^{15}$ However, the bone resection is extended ventrally via a transpedicular decancellization of the C-7 VB. The osseous removal targets the anterior longitudinal ligament and moves medially until the cavity communicates with the contralateral side. The lateral walls of the VB are resected in a wedge V-shaped manner. The remaining posterior vertebral wall is pushed into the decancellized space and removed piecemeal. The decancellized osteotomy is then closed, with the correction occurring about an internal axis of rotation centered over the anterior longitudinal ligament. Correction is performed using a smooth controlled maneuver, without the sudden fracture associated with an extension osteotomy. Additional benefits include the optimized fusion environment of 2 apposed cancellous surfaces, and the overall spinal shortening procedure, which reduces stretching of anterior structures such as the trachea and esophagus.

\section{Anterior Release and Anterior Osteotomy}

Several authors have reported performing an initial anterior release prior to the posterior extension osteotomy $y^{3,4,12}$ Mummaneni and coworkers ${ }^{12}$ have described a staged anterior-posterior-anterior procedure for cervical osteotomy at C5-6. The initial approach is through an anterior osteotomy with the patient positioned supine. The anterior procedure consists of a C5-6 discectomy and resection of a dorsal VB wedge by undercutting the inferoposterior edge of $\mathrm{C}-5$ and the superoposterior edge of C- 6 . The second stage of the procedure is a posterior osteotomy performed similarly to that described by Urist ${ }^{19}$ and Simmons,${ }^{15}$ but with the patient positioned prone. The deformity is again corrected by manually extending the neck. Because of the anterior osteotomy and release, the corrective maneuver is performed in a controlled motion. The posterior closing osteotomy is supplemented with internal fixation via a screw/rod construct. For the final stage of the operation, the patient is repositioned supine, the anterior exposure is reopened, and a tricortical iliac autograft is placed in the anterior opening wedge defect. A cervical plate and screws are placed for anterior fixation.

The anterior release and osteotomy provide several benefits. With an anterior osteotomy, the internal axis of rotation is shifted ventrally in the $\mathrm{VB}$, rather than the posterior longitudinal ligament. This allows for controlled correction with neck extension, without the abrupt fracture that occurs with osteoclasis. Also, the surgeon determines the level of the anterior wedge osteotomy rather than an extension osteoclasis, which may cause fracture at a random, undesired level. An anterior approach also allows placement of 
a structural graft in the anterior opening wedge defect (Fig. 7). The necessity of an anterior release, however, is debatable because an ankylosed, osteoporotic spine generally fractures without requiring excess manual force. ${ }^{9}$ Performing an initial anterior procedure in patients with cervical kyphosis is technically challenging, particularly in those with a chin-on-chest deformity, in whom the available corridor of access and working trajectory are significantly compromised. In extreme cases, anterior exposure may not be possible. Once the anterior spine is exposed, differentiating the disc space from the VB is complicated by progressive syndesmophyte formation, which obscures the normal anatomical landmarks. With staged procedures, one must consider the risks associated with an extended anesthesia time, as well as multiple repositioning of the patient.

\section{Level of Osteotomy}

Overwhelmingly, the level of osteotomy and correction for cervical flexion deformity described in the literature is performed at C7-T1. ${ }^{1,7,9,11,13,14,19}$ The benefits of performing the osteotomy at the cervicothoracic junction include a wider spinal canal, sparing of upper-extremity function in event of a cord injury, and vertebral artery anatomy relative to C-7. Posterior extension osteotomy has been performed, however, at midcervical levels. ${ }^{6}, 12$ In cases of extreme kyphosis, performing the osteotomy at the apex of the maximum curvature may allow for a more anatomical correction. ${ }^{6}$ Because the vertebral artery enters the transverse foramen at C-6, an extension osteotomy above C-7 requires exposing bilateral vertebral arteries from their respective transverse foramen to prevent kinking after correction. ${ }^{12}$ Releasing the vertebral artery from the osseous confines of the transverse foramen can be performed from a combined anterior-posterior exposure. ${ }^{12}$

\section{Procedural Complications}

Cervical flexion deformity in AS is challenging. Likewise, surgical correction involving an extension osteotomy is a technically demanding procedure. As a result, there is only a small body of literature covering extension osteotomy for cervical deformity in AS, with the majority being small series or case reports. Reviewing all series with at least 10 patients with AS who underwent extension osteotomy, ${ }^{1,7,9,14}$ we found that 5 of 183 patients overall had significant spinal cord injury. This included 1 case of paraparesis, 1 case of hemiparesis, and 3 cases of tetraparesis. There were 6 reported deaths in the postoperative period (within 3 months). Minor neurological complications included several cases of transient postoperative weakness with spontaneous recovery. There were 35 cases of C-8 sensory disturbances, with the majority resolving within weeks to months. In certain instances, patients were immobilized in a halo vest without internal fixation underwent gentle distraction of the halo to relieve foraminal compression of the C-8 nerve root. Other complications included transient postoperative dysphagia that generally resolved within weeks. ${ }^{1,9}$ Pseudarthrosis was a rare complication that may be related to delayed subluxation at the osteotomy site. ${ }^{1}$ Simmons et al. ${ }^{14}$ reported 6 nonunions occurring in
131 patients, and McMaster ${ }^{9}$ found 2 cases of pseudarthrosis in 15 patients. In both series, the patients in whom nonunions developed were treated without internal fixation.

\section{Conclusions}

Correcting cervical flexion deformity in patients with AS is challenging and carries potential risk for serious neurological injury. Careful patient selection and meticulous preoperative planning are essential to counterbalance these risks. Important considerations in presurgical decision making include choosing an appropriate anesthetic modality, neuromonitoring, patient positioning, surgical approach, and spinal instrumentation. We recommend the use of general anesthesia with MEP and SSEP electrophysiological monitoring. Modern anesthestic techniques allow for safe fiberoptic intubation, which maintains a secure airway and ensures patient comfort during the procedure. General anesthesia also allows for the patient to be positioned prone. The prone position provides access to the full cervical and thoracic spine for multilevel decompression, if necessary, and placement of a segmental internal fixation device. Long-segment screw/rod constructs offer better stabilization compared with halo immobilization alone, thereby reducing the risk of postoperative subluxation or implant failure.

Successful correction of a cervical kyphosis can generally be performed via a single posterior approach for osteotomy, reduction, and stabilization. A combined anterior-posterior approach may be necessary in certain circumstances. Anterior procedures should be performed in patients with ventral compression of the spinal cord. Patients with severely osteoporotic bone and increased risk for failure of posterior screws may require reconstruction of the anterior column defect and supplemental anterior fixation. Otherwise, a single posterior procedure limits the operative time, duration of anesthesia, and patient morbidity.

In conclusion, cervical kyphosis resulting in impaired forward vision, swallowing, hygiene, and social outlook can lead to significant disability. Cervical extension osteotomy remains the primary treatment modality and, although technically demanding, can significantly improve patient psychological and functional outcome.

\section{References}

1. Belanger TA, Milam RA IV, Roh JS, Bohlman HH: Cervicothoracic extension osteotomy for chin-on-chest deformity in ankylosing spondylitis. J Bone Joint Surg Am 87:1732-1738, 2005

2. Ben-David B, Haller G, Taylor P: Anterior spinal fusion complicated by paraplegia. A case report of a false-negative somatosensory-evoked potential. Spine 12:536-539, 1987

3. Bhojraj SY, Dasgupta D, Dewoolkar LV: One-stage "front" and "back" correction for rigid cervical kyphosis. A safer technique of correction for a rare case of adult-onset Still's disease. Spine 18:1904-1908, 1993

4. Bouchard JA, Feibel RJ: Gradual multiplanar cervical osteotomy to correct kyphotic ankylosing spondylitic deformities. Can J Surg 45:215-218, 2002

5. Cornefjord M, Alemany M, Olerud C: Posterior fixation of subaxial cervical spine fractures in patients with ankylosing spondylitis. Eur Spine J 14:401-408, 2005

6. Duff SE, Grundy PL, Gill SS: New approach to cervical flexion 
deformity in ankylosing spondylitis. Case report. J Neurosurg 93 (2 Suppl):283-286, 2000

7. Langeloo DD, Journee HL, Pavlov PW, de Kleuver M: Cervical osteotomy in ankylosing spondylitis: evaluation of new developments. Eur Spine J 15:493-500, 2006

8. Lesser RP, Raudzens P, Lüders H, Nuwer MR, Goldie WD, Morris HH III, et al: Postoperative neurological deficits may occur despite unchanged intraoperative somatosensory evoked potentials. Ann Neurol 19:22-25, 1986

9. McMaster MJ: Osteotomy of the cervical spine in ankylosing spondylitis. J Bone Joint Surg Br 79:197-203, 1997

10. Mehdian H, Jaffray D, Eisenstein S: Correction of severe cervical kyphosis in ankylosing spondylitis by traction. Spine 17: 237-240, 1992

11. Mehdian SM, Freeman BJ, Licina P: Cervical osteotomy for ankylosing spondylitis: an innovative variation on an existing technique. Eur Spine J 8:505-509, 1999

12. Mummaneni PV, Mummaneni VP, Haid RW Jr, Rodts GE Jr, Sasso RC: Cervical osteotomy for the correction of chin-on-chest deformity in ankylosing spondylitis. Technical note. Neurosurg Focus 14(1): E9, 2003

13. Shimizu K, Matsushita M, Fujibayashi S, Toguchida J, Ido K, Nakamura T: Correction of kyphotic deformity of the cervical spine in ankylosing spondylitis using general anesthesia and internal fixation. J Spinal Disord 9:540-543, 1996
14. Simmons ED, DiStefano RJ, Zheng Y, Simmons EH: Thirty-six years experience of cervical extension osteotomy in ankylosing spondylitis: techniques and outcomes. Spine 31:3006-3012, 2006

15. Simmons EH: Kyphotic deformity of the spine in ankylosing spondylitis. Clin Orthop Relat Res 128:65-77, 1977

16. Smith-Petersen MN, Larson CB, Aufranc OE: Osteotomy of the spine for correction of flexion deformity in rheumatoid arthritis. Clin Orthop Relat Res 66:6-9, 1969

17. Taggard DA, Traynelis VC: Management of cervical spinal fractures in ankylosing spondylitis with posterior fixation. Spine 25: 2035-2039, 2000

18. Tokala DP, Lam KS, Freeman BJ, Webb JK: C7 decancellisation closing wedge osteotomy for the correction of fixed cervico-thoracic kyphosis. Eur Spine J 16:1471-1478, 2007

19. Urist MR: Osteotomy of the cervical spine; report of a case of ankylosing rheumatoid spondylitis. J Bone Joint Surg Am 40: 833-843, 1958

Manuscript submitted October 13, 2007.

Accepted November 7, 2007.

Address correspondence to: Michael Y. Wang, M.D., Department of Neurological Surgery University of Miami, Miller School of Medicine, Lois Pope Life Center, 1095 NW 14th Terrace, Miami, Florida 33136. email: mwang2@med.miami.edu. 